\title{
Reverse k-skyband query based on reuse technology
}

\author{
Tao Jiang", Bin Zhang ${ }^{\#}$ \\ ${ }^{\#}$ College of Mathematics Physics and Information \\ Engineering \\ Jiaxing University \\ Jiaxing, P. R. China \\ e-mail: jiangtao_guido@yahoo.com.cn
}

\author{
Lu Chen ${ }^{*}$, Qing Liu*, Jianyong Yan \\ ${ }^{*}$ College of Computer Science and Technology \\ Zhejiang University \\ Hangzhou, P. R. China \\ e-mail: \{luchen, qingliu\}@ zju.edu.cn
}

\begin{abstract}
In this paper, we introduce a new approach to finish reverse $k$-skyband (RkSB) query which returns all the points in given dataset $P$ whose dynamic $k$-skyband contains specific query object q. The main ideas include reuse technology and early stopping. The former save the information of node accesses during $R$-tree search into an auxiliary heap so that dramatically decreases the I/O cost and improves the CPU efficiency. This is because RkSB needs to execute global $k$-skyband and window query to finish the computation and the reuse information can be used in the phase of window query. The latter improves the efficiency of RkSB computation. The experimental results conducted on real datasets and synthetic datasets show that our proposed algorithm is effective and has a better performance both $I / O$ cost and CPU efficiency than the algorithm based on reverse BBS (Branch and Bound Skyline) method.
\end{abstract}

Keywords- algorithm; skyline query; reverse skyline; skyband

\section{INTRODUCTION}

Recently, many researchers from database research community pay much attention to skyline queries [1-8] due to its wide applications related to multi-criteria decision making. Specifically, given a d-dimensional data set $D$, a skyline query [3] retrieves all the data objects which are not dominated by other objects in $D$. A data object $p$ dominates another data object $p^{\prime}$ if $p$ is not worse than $p^{\prime}$ in all dimensions and strictly better than $p^{\prime}$ in at least one dimension.

Papadias et al. [3] relax the restriction of skyline definition and extend skyline queries to $k$-skyband queries, which return all the data objects that are dynamically dominated by at most $k$ data objects. In fact, to identify the influence of query object $q$, traditional skyline queries can be computed from company perspective. This skyline query is called reverse skyline query [6-7]. In contrast, reverse $k$ skyband (RkSB) query proposed by Liu et al. [4] is a natural extension of dynamic $k$-skyband query. Although RkSB query can be answered by the algorithms in [4], for example, branch-bound-based RkSB (BRkSB), they do not take advantage of reuse technology. In fact, $\mathrm{R} k \mathrm{SB}$ produces many redundant $\mathrm{I} / \mathrm{O}$ owing to the window query during the procedure of search.

In this paper, we propose a novel RkSB algorithm which utilizes reuse information technology [8] and early stopping method. We call the algorithm RRkSB (reuse RkSB). Our experimental results show that RRkSB reduces more than
90\% I/O cost than BR $k \mathrm{SB}$; meanwhile, $\mathrm{RR} k \mathrm{SB}$ has a less $\mathrm{CPU}$ time than $\mathrm{BR} k \mathrm{SB}$ in most of cases.

\section{PROBLEM FORMULATION}

In this section, we illustrate the definitions of dynamic $k$ skyband, global $k$-skyband, and reverse $k$-skyband.

Definition 1 (Dynamic $k$-Skyband, DkSB). Given a multiple dimensional data set $D$, a query object $q$, and a parameter $k$, if a data object $p \in D$ belongs to the dynamic $k$ skyband of $q$, there exist at most $k$ data objects in $D$ so that they dynamically dominate $p$ with respect to $q$.

Consider the example in Figure 1(a), where the data set is $D=\left\{p_{1}, p_{2}, \ldots, p_{8}\right\}$ and $p_{1}{ }^{\prime}$ and $p_{6}{ }^{\prime}$ are the projections of $p_{1}$ and $p_{6}$ with regard to $p_{2}$. The results of 1-skyband of $p_{2}$ are the set $R^{\prime}=\left\{p_{3}, p_{5}, p_{1}, p_{4}, q, p_{6}\right\}$. For instance, there no data objects dominating object $p_{3}$ and $p_{4}$ is only dominated by $p_{3}$.

Definition 2 (Reverse $k$-Skyband, RkSB). Given a multiple dimensional data set $D$, a query object $q$, and a parameter $k$, the reverse $k$-skyband query of $q$, denote as $\operatorname{RkSB}(q)$, returns all the data objects in $D$ whose dynamic $k$ skyband contains $q$.

Essentially, parameter $k$ represents the thickness of skyline. When $k$ becomes zero, the skyline corresponds to original skyline. For example, the results of $\operatorname{DoSB}\left(p_{2}\right)$ are $\left\{p_{3}, p_{5}\right\}$ in Figure 1(a), and $\operatorname{ROSB}(q)$ in Figure 1(b) returns $\left\{p_{3}, p_{6}, p_{8}\right\}$ as the results; however, when $k$ becomes 1 , $\operatorname{D1SB}\left(p_{2}\right)$ in Figure $1(\mathrm{a})$ retrieves the set of $\left\{p_{3}, p_{5}, p_{1}, p_{4}, q\right.$, $\left.p_{6}\right\}$ and $\operatorname{R} 1 \mathrm{SB}(q)$ in Figure $1(\mathrm{~b})$ returns the set of $\left\{p_{3}, p_{4}, p_{2}\right.$, $\left.p_{5}, p_{7}, p_{9}, p_{6}, p_{8}\right\}$ as the results.

Definition 3 (Global $k$-Skyband, GkSB). Given a query object $q$, a $d$-dimensional data set $D$, and a parameter $k$, if a data object $p \in D$ is in the global $k$-skyband of $q$, there exist at most $k$ data objects in $D$ which globally dominate [7] $p$.

GkSB query runs a window query to judge whether the current object $p$ is in global $k$-skyband of $q$. Consider the example in Figure 1(b), the window of $p_{5}$ corresponds to the rectangle area, $w\left(p_{5}, q\right)$, which centers on $q$. Because $w\left(p_{5}, q\right)$ only includes a data object $p_{2}$, the object $p_{5}$ is among global $k$-skyband of $q$. Similarly, $p_{1}$ is not in global $k$-skyband of $q$ because $w\left(p_{1}, q\right)$ contains two other data objects $p_{3}$ and $p_{4}$. In Figure 1(b), the data objects among the gray areas consists of the results of $\operatorname{D1SB}(q)$ among partitions I, II, III, and IV, namely, $\left\{p_{1}, p_{3}, p_{4}\right\},\left\{p_{7}, p_{9}\right\},\left\{p_{2}, p_{5}\right\}$ and $\left\{p_{6}, p_{8}\right\}$. 


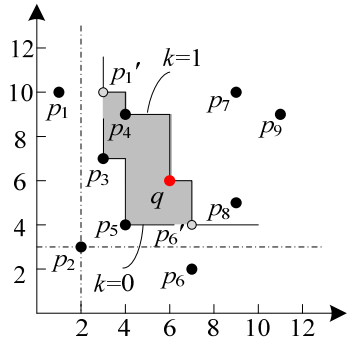

(a)

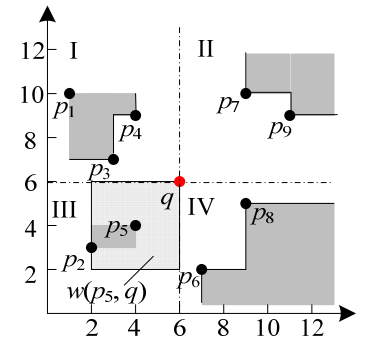

(b)
Figure 1. Illustration of $k$-skyband. (a) 1-skyband of $p_{2}$, (b) reverse 1 skyband of $q$.

\section{RKSB QUERY PROCESSING}

In this section, we firstly introduce how to reduce redundant $\mathrm{I} / \mathrm{O}$ cost, and then give the procedure of query processing of our proposed RRkSB algorithm.

\section{A. Reduce redundant $I / O$ cost}

Clearly, naïve RkSB query implemented by definition 2 has $O(|D| \times \operatorname{DkSB}(q))$ time complexity. Owing to a larger cardinality of dataset $D$, the algorithm has a lowest efficiency. Recently, Liu et al. [4] introduces a better algorithm, called BRkSB. Because BRkSB is a customized algorithm of branch and bound skyline [3], it has optimal I/O performance.

However, BRkSB still has many redundant I/O accesses as a result of not reusing the node information of heap during the GkSB query. Consider the example in Figure 2, if the node information after G1SB query of $q$ has been executed, are saved in a reuse heap $H_{r}$, i.e., the nodes of $N_{5}, N_{1}$, and $N_{6}$, the execution of G1SB of $q$ can avoid to access root node. In fact, each window query of GkSB needs to access R-tree [9] one time. Therefore, this will bring many redundant $\mathrm{I} / \mathrm{O}$ cost. This is because RkSB query cannot use the window query based on memory.

Now, let us consider the procedure of G1SB of $q$. R $k$ SB requires executing multiple window queries for data objects, $b, c, d, e, f, g, h$, and $i$. For the window query of $c$, the root node is firstly accessed; then the node $N_{1}$ is accessed since it is intersected with the window $w(c, q)$; finally, the node $N_{3}$ is accessed. In fact, only one access is necessary and two redundant $\mathrm{I} / \mathrm{O}$ accessed can be avoided because the G1SB of the first object $b$ has saved the node information of root and $N_{1}$ into the reuse heap. Similarly, the window query of $g$ brings 1 redundant $\mathrm{I} / \mathrm{O}$ accesses since the root node is contained in reuse heap.

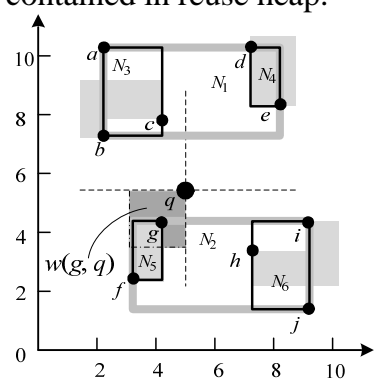

(a)

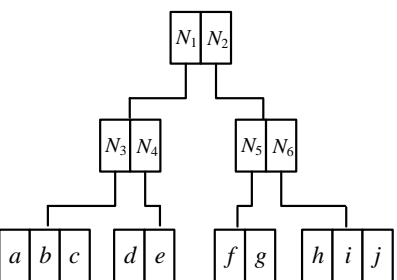

(b)
Figure 2. An example of R-tree index for reverse $k$-skyband query. (a) G1SB of $q$, (b) the structure of R-tree.
Therefore, a method to reduce redundant $\mathrm{I} / \mathrm{O}$ is maintaining the node information having been accessed according to the procedure of RkSB search. We use a reuse heap to save the node information so that next window query can use them. Thus, much redundant $\mathrm{I} / \mathrm{O}$ cost is saved. We call the algorithm reuse RkSB (RRkSB) algorithm. But, note that the mechanism of $\mathrm{RR} k \mathrm{SB}$ is different from cache method because the former is related with $\mathrm{R} k \mathrm{SB}$ procedure and the latter is related with local node information. Specifically, RRkSB inserts the node having been accessed into the reuse heap. When the child node of some node needs to be accessed, RRkSB extends the node and then deletes it from reuse heap.

\section{B. Query Processing}

In this subsection, we discuss the detailed RR $k$ SB query processing which searches the R-tree [9] index in best first (BF) manner [3], and, meanwhile, utilizes the reuse heap to save the node information being accessed. Algorithm 1 illustrates the RRkSB query procedure in detail. Specifically, we first initialize the set variable $S_{r}$ to the query results and the set variable $S_{c}$ to save the candidate object (i.e., global $k$ skyband objects) at line 1 , and then maintain a minimum heap $H$ with entries in the form (e,key) (line 2), where $e$ is an MBR node of index and key is defined as the minimum between $q$ and $e$. Every time we pop out an entry ( $e$, key) from $H$ with the minimum key value (line 4). Then, we verify whether or not $e$ is globally dominated by $k+1$ objects in $S_{\text {c }}$. If it is true, then $e$ can be safely pruned; otherwise, we process $e$ as follows.

If $e$ is an intermediate node, we check each child $e_{i}$ of $e$ to judge whether it is globally dominated by $k+1$ objects in $S_{\mathrm{c}}$ (line 8). If it is true, we discard $e_{i}$; otherwise, we insert $e_{i}$ into auxiliary heap $H$ (line 9). Next, we update the reuse heap $H_{r}$ at line 10 . Note that the line is very important. It guarantees that all nodes accesses are saved in $H_{r}$ and there are no redundant nodes. In order to shrink the capacity of $H_{r}$, we might only insert intermediate node into $H_{r}$. In addition, in order to improve the efficiency of search, we adopt the binary search method. If $e$ is a data object, we insert $e$ into $S_{c}$ which is a candidate set to save global $k$-skyband objects (line 12). Then, we invoke $k$-WindowQuery procedure at line 13 to judge whether or not the window $w(e, q)$ contains more than $k$ other data objects. If it is true, we mark $e$ as false alarm by line 15; otherwise, $e$ will be returned as query results at line 16 .

Algorithm 1. $\operatorname{RRkSB}\left(T, q, k, S_{r}\right)$

Input: $T$-index; q-query object

Output: $S_{r}-$ result set

1. $S_{r}=\varnothing, S_{c}=\varnothing, H=\varnothing, H_{r}=\varnothing$

2. Insert all entries of root into $H, H_{r}$ with $(e, \operatorname{mindist}(e, q))$

3. WHILE $H$ is not empty DO

4. Pop the entry $(e$, mindist $(e, q))$ of $H$

5. IF $e$ is not globally dominated by $(k+1)$ points in $S_{c}$

6. IF $e$ is an intermediate entry THEN

7. FOR each child entry $e_{i} \in e$ DO

8. IF $e_{i}$ isn't globally dominated by $(k+1)$ points in $S_{c}$

9. $\quad$ Insert $\left(e_{i}\right.$, minidist $\left.\left(e_{i}, q\right)\right)$ into $H$

10. Insert all $e_{i} \in e$ into $H_{r}$, Delete $e$ from $H_{r}$

11. ELSE //e is a data object 
12. $S_{c}=S_{c} \cup\{e\} / /$ insert $e$ into global $k$-skyband

13. $\quad r$ flag $=\boldsymbol{k}$-WindowQuery $\left(T, e, q, H_{r}\right)$

14. IF $r$ flag==TRUE THEN//contain $(>k+1)$ other objects

15. Mark $e$ as false alarm

16. RETURN all not false alarm points in $S_{r}$

The window query procedure, namely, $k$-WindowQuery, is described in detail as algorithm 2. It first initializes a auxiliary heap $H$ and a set variable $S_{w}$ which is used to save the objects in window $w(p, q)$ at line 1 , and then inserts all entries which intersects with $w(p, q)$ into $H$ by line 2 . Next, algorithm 2 uses best first search policy to obtain all objects in window $w(p, q)$ and inserts them into $S_{w}$. When the number of objects in $S_{w}$ is great than $k$, algorithm 2 returns FALSE; otherwise, it return TRUE until algorithm 2 finishes the search (line 14). Lines 10-13 extends the intermediate node and inserts all child entries which is inside or overlap with $w(p, q)$ into $H$. But, note that the key in algorithm 2 becomes the distance between $p$ and current $e$, namely, minidist $(e, p)$. Since algorithm 2 can stop the search, it does not has to search all nodes in index and obtain a higher efficiency.

Algorithm 2. $k$-WindowQuery $\left(p, q, H_{r}, k\right)$

Input: $p$-current object; $q$-query object; $H_{r}$-reuse heap, $k$-user specified parameter

Output: a Boolean value to indicate whether the current window $w(p, q)$ contains more than $k$ objects

1. $H=\varnothing, S_{w}=\varnothing$, get query window $w q(p, q)$

2. insert entries of $H_{r}$ which intersect with $w(p, q)$ into $H$ with the form $(e$, minidist $(e, p))$

3. WHILE $H$ is not empty DO

4. de-heap the top entry $(e$, mindist $(e, p))$ from $H$

5. IF $e$ is a data object THEN

6. IF $e \neq p$ and $e$ is contained in $w q(p, q)$ THEN

7. insert $e$ into $S_{w} / / S_{w}$ saves the objects in $w(p, q)$

8. IF $\left|S_{w}\right|>k$ THEN RETURN FALSE

9. ELSE //intermediate entry

10. IF $w q(p, q)$ is inside or overlap with $e$ THEN

11. FOR each child entry $e_{i} \in e$ DO

12. IF $w q(p, q)$ is inside or overlap with $e_{i}$ THEN

13. insert the entry $\left(e_{i}\right.$, minidist $\left.\left(e_{i}, p\right)\right)$ into $H$

14. RETURN TRUE

By above analysis, now we immediately obtain the following important Theorem 1 and Theorem 2 .

Theorem 1. For a given query object $q$, RRkSB algorithm correctly returns all results of reverse $k$-skyband, and does not product false alarms or false dismissals.

Proof. According to Definition 2 and Definition 3, we know that the candidate set of global $k$-skyband, Sc, will correctly save the results of global $k$-skyband because RRkSB uses the method of global dominating at line 5 and line 8. On the other hand, it is equivalent to obtain the node information by using reuse heap $H_{r}$ and accessing index. For example, in Figure 2, $N_{5}$ corresponds to data objects $f$ and $g$, $N_{1}$ corresponds to data objects $a, b, c, d$, and $e$, and $N_{5}$ corresponds to data objects $h, i$, and $j$ when the nodes among $H_{r}$ are $N_{5}, N_{1}$, and $N_{6}$. Thus, the procedure of $k$-WindowQuery and the window query of $k$-skyband have the same effect. Therefore, Theorem 1 is correct.

Theorem 2. For the entries in reuse heap $H_{r}$, BRkSB need to access multiple times disk, yet RRkSB only accesses disk one time.

Proof. According to the process of reverse $k$-skyband, we know that it is divided two stages, namely, global $k$-skyband and $k$-window query. Since the nodes among reuse heap $H_{r}$ are inserted after the first access, RRkSB can directly fetch these nodes from $H_{r}$ when they are accessed later. Thus, RRkSB only need to access disk one time. However, BRkSB will access these nodes in $H_{r}$ at both the stage of global $k$ skyband and the stage of $k$-window query. Therefore, $\mathrm{BR} k \mathrm{SB}$ requires at least two disk accesses. For instance, consider the example in Figure 2, $H_{r}$ becomes $g, N_{1}, N_{6}$, and $f$ when RRkSB obtains the first object of global 1-skyband, $g$. Then, the window queries of the objects $b, c, d$ and $e$ require to access node $N_{1}$ one time, respectively. Consequently, BRkSB produces $5 \mathrm{I} / \mathrm{O}$ accesses. So, Theorem 2 is correct.

\section{EXPERIMENTAL EVALUATION}

In this section, we experimentally evaluate the performance of BRkSB [4] and RR $k$ SB algorithms for R $k \mathrm{SB}$ retrieval over both real and synthetic datasets. The real dataset Color $(\mathrm{Col})$ includes $3 \mathrm{D}$ vectors representing the color histograms of $68 \mathrm{~K}$ images [5]. We also create a 3D synthetic dataset Correlated (Corr) used in traditional skyline. The values of the attributes are in the range [1, 10000]. In each experiment we performed 50 reverse skyline queries to the particular data set and reported the overall result. Each dataset is indexed by an R-tree, where the page size is set to $4 \mathrm{~KB}$ in all cases. All algorithms were implemented in $\mathrm{C}++$, and all experiments were conducted on an Intel Dual Core 2.0 GHz PC with 4GB RAM.

\section{A. The Effect of parameter $k$}

The first set of experiments studies the performance of proposed algorithms as the function of parameter $k$. Figure 3 shows the results. With the increase of $k$, the I/O cost quickly grows, but CPU time only slowly increases. This is because there are more query results when the parameter $k$ increases. In most of cases, the I/O cost of RR $k S B$ is $1 / 5$ that of BRkSB and CPU time of RRkSB is about $3 / 5$ that of BR $k S B$. This indicates that RR $k$ SB has a better performance than BR $k$ SB on parameter $k$.

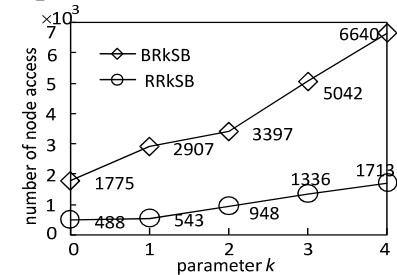

(a)

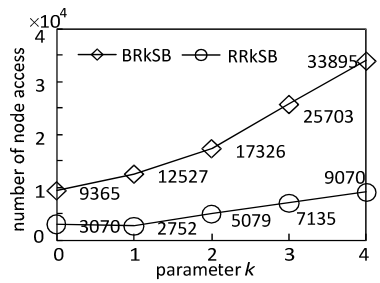

(c)

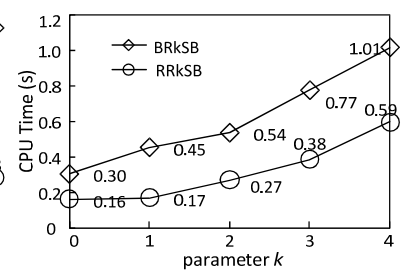

(b)

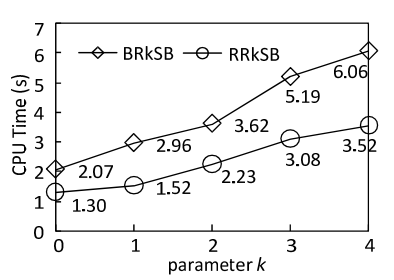

(d) 
Figure 3. The comparison of the performance for the algorithms vs. $k$. (a) I/O cost on Col, (b) CPU Time on Col, (c) I/O cost on Corr, (d) CPU Time on Corr.

\section{B. The Effect of dataset size}

The second set of experiments reports the influence of dataset size on the algorithms, varying the cardinality of $\mathrm{Col}$ from $12 \mathrm{~K}$ to $60 \mathrm{~K}$, the cardinality of Corr from $128 \mathrm{~K}$ to 2048K, respectively. Figure 4 gives the results. Although the cost of the algorithms both grows with the increase the size of dataset, the cost on $\mathrm{Col}$ has a slower raise than that on Corr. The reason lies in the different distribution of two datasets. Whichever dataset is used, RRkSB gains better performance than $\mathrm{BR} k \mathrm{SB}$.

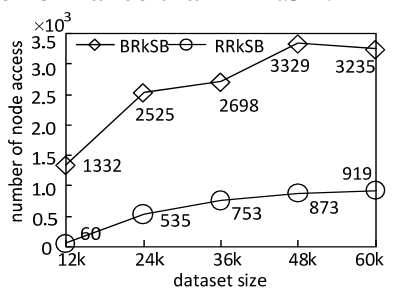

(a)

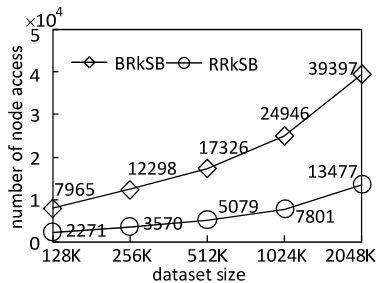

(c)

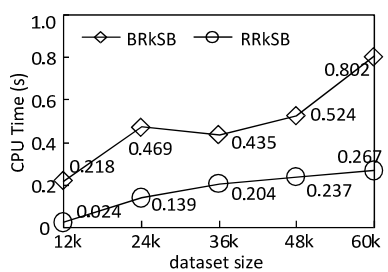

(b)

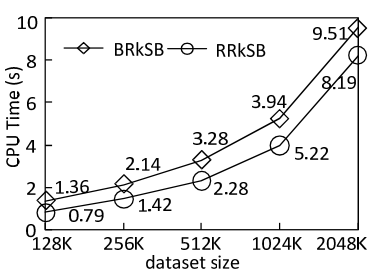

(d)
Figure 4. The comparison of the performance for the algorithms vs. dataset size. (a) I/O cost on Col, (b) CPU Time on Col, (c) I/O cost on Corr, (d) CPU Time on Corr.

\section{The Effect of cache size}

The third serial of experiments aims to evaluate the effect of cache size on the algorithms. We change the number of cache blocks from 0 to 4, where each block contains 4096 bytes. Figure 5 plots the cost of the algorithms w.r.t. the number of cache blocks. Clearly, we find that the I/O cost slowly decreases with the increase of the number of cache blocks; yet there is no change on CPU time over two datasets. This verifies the usefulness of our proposed reuse technology. The reason lies that cache cannot forecast next cache block used by the algorithms, and reuse method save the next block into reuse heap. Comprehensively, $\mathrm{RR} k \mathrm{SB}$ obtains better performance than BR $k \mathrm{SB}$. (a)

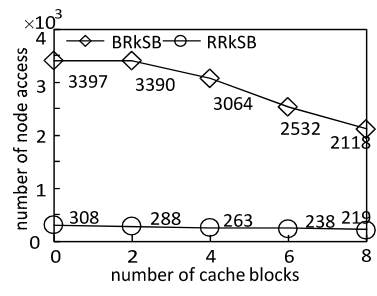

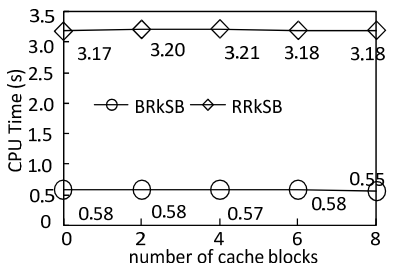

(b)

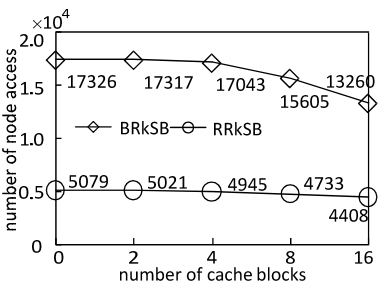

(c)

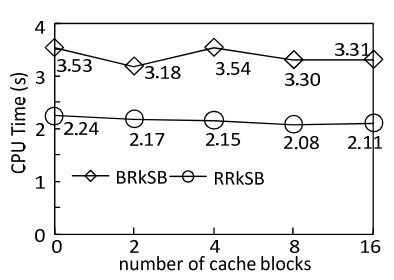

(d)
Figure 5. The comparison of the performance for the algorithms vs. the number of cache blocks. (a) I/O cost on Col, (b) CPU Time on Col, (c) I/O cost on Corr, (d) CPU Time on Corr.

\section{CONCLUSION}

This paper introduces a novel algorithm to answer the reverse $k$-Skyband query, namely, reuse $\mathrm{R} k \mathrm{SB}$ (RR $k \mathrm{SB})$. The main ideas are reuse information technology and early stopping computation. The experiments conducted on real and synthetic datasets demonstrate the efficiency and effectiveness of RRkSB, compared with basic RkSB algorithm (BRkSB) [4].

\section{ACKNOWLEDGMENT}

This work was supported by NSFC 61003049, the Research Innovation Team Funds for College Students of Zhejiang Province, ZJNSF LY12F02047 and LY12F02019, 2011AY1005, the Fundamental Research Funds for the Central Universities under grant 2010QNA5051.

\section{REFERENCES}

[1] I. Bartolini, Z. Zhang, and D. Papadias, "Collaborative Filtering with Personalized Skylines,” IEEE Trans. Knowl. Data Eng., vol. 23, Feb 2011, pp. 190-203, doi: 10.1109/TKDE.2010.86

[2] K. Hose and A. Vlachou, "A Survey of Skyline Processing in Highly Distributed Environments,” The VLDB Journal, vo. 21, June 2012, pp. 359-384, doi: 10.1007/s00778-011-0246-6.

[3] D. Papadias, Y. Tao, G. Fu, and B. Seeger, "Progressive Skyline Computation in Database Systems,” ACM Trans. on Database Syst., vol. 30, March 2005, pp. 41-82, doi: 10.1145/1061318.1061320.

[4] Q. Liu, Y. Gao, G. Chen, and Q. Li, "On Efficient Reverse k-Skyband Query Processing,” Proc. of DASFAA Conf., Springer, April 2012, pp. 544-559, doi: 10.1007/978-3-642-29038-1_39.

[5] Y. Tao, X. Xiao, and J. Pei, "Efficient Skyline and Top- $k$ Retrieval in Subspaces,” IEEE Trans. Knowl. Data Eng., vol. 19, Aug. 2007, pp. 1072-1088, doi: 10.1109/TKDE.2007.1051.

[6] E. Dellis and B. Seeger, "Efficient Computation of Reverse Skyline Queries,” Proc. of VLDB Conf. (VLDB07), VLDB Endowment, Sep. 2007. pp. 291-302.

[7] L. Chen and X. Lian, "Monochromatic and Bichromatic Reverse Skyline Search over Uncertain Databases,” Proc. of ACM SIGMOD, ACM Press, June. 2008. pp. 213-226, doi: 10.1145/1376616.1376641.

[8] J. Lee and S. Hwang, "QSkycube: Efficient Skycube Computation using Point-Based Space Partitioning," Proc. of the VLDB Endowment, vo. 4, Dec. 2010, pp. 185-196.

[9] A. Guttman, "R-Trees: A Dynamic Index Structure for Spatial Searching,” ACM SIGMOD Record, vol. 14, June 1984, pp. 47-57, doi:10.1145/971697.602266. 